\title{
Prácticas de publicación en campos disciplinares percibidas por investigadores de una universidad pública mexicana
}

\section{Publication practices in discipline-oriented fields as perceived by researchers in a public university in Mexico}

DOI: https://doi.org/10.32870/dse.v0i23.765

\author{
Margarita Flor Méndez-Ochaita* \\ Alma Carrasco Altamirano** \\ Mara Serrano Acuña***
}

\begin{abstract}
Resumen
Este artículo revisa la publicación como práctica científica de comunidades disciplinares a través de un estudio empírico y exploratorio con 352 investigadores de una universidad pública estatal mexicana. Se aplicó una encuesta en línea para conocer las percepciones de los investigadores sobre el quehacer científico en sus campos disciplinares. En este trabajo se analizan las respuestas a los ítems relacionados con las prácticas de publicación (seis de un total de 41 ítems del instrumento). El enfoque es cualitativo y la interpretación se fundamenta en los aportes clásicos de la sociología de la ciencia de Robert Merton sobre la estructura normativa que regula la actividad científica. Los resultados sugieren que entornos institucionales de exigencia para la producción académica en las disciplinas alimentan prácticas científicas variadas. La mayoría de investigadores percibe que en sus campos disciplinares se aprovecha tanto el conocimiento producido localmente como el extranjero, pero en todas las disciplinas hay una parte de la comunidad científica que percibe prácticas que están en tensión con las normas mertonianas, tales como: interesarse más por adquirir fama personal que por ampliar la base de conocimiento, incurrir en plagio, fragmentar de manera intencionada una investigación para construir varias publicaciones, y ejercer el rol de dictaminador anclado a creencias y opiniones personales por encima de valores universales de construcción del conocimiento científico. Esto tiene implicaciones para la formación de los aprendices de científicos que en
\end{abstract}

* Doctora en Ciencias, especialidad de Investigaciones Educativas. Profesora y coordinadora de la Maestría en Estudios del Discurso y Literacidades Académicas de la Universidad Autónoma de Tlaxcala. Líneas de investigación: formación de investigadores y las prácticas científicas en educación superior, estudio de nuevas literacidades en la formación de posgrado. Universidad Autónoma de Tlaxcala. México. flormendezo@yahoo.com

* Psicóloga con estudios en Ciencias del Lenguaje, doctora en Educación. Académica de la Benemérita Universidad Autónoma de Puebla (BUAP). Presidenta Fundadora del Consejo Puebla de Lectura, A.C. Líneas de investigación: fomento de lectura y bibliotecas; leer en primera infancia; trayectorias formativas de científicos y autoría; lenguaje y currículo. México. almacarrascoa@gmail.com

*** Doctora en Educación. Profesora de tiempo completo. Miembro del Padrón de Investigadores y coordinadora del área de tecnología aplicada al diseño gráfico, Benemérita Universidad Autónoma de Puebla (BUAP). Miembro de la Red Internacional de Investigación en Ciencias Sociales Interdisciplinares de Common Ground Research Networks 2019, con reconocimiento del Programa para el Desarrollo Profesional Docente, para el Tipo Superior. México. maraserrano@gmail.com 
el nivel de posgrado participan de las prácticas de los especialistas y se están preparando para escribir y publicar géneros textuales especializados como el artículo de investigación.

Palabras clave: comunidades disciplinares - literacidad científica - ethos científico - roles - prácticas científicas.

\section{Abstract}

This article reviews scientific publication as a practice across academic discipline-oriented communities through an empirical and exploratory study with 352 researchers from a Mexican public state university. An online survey was applied to learn how researchers perceived their scientific labor in their academic disciplinary fields. Answers to items related to publication practices were analyzed ( 6 from a total of 41 items). Using a qualitative approach, we interpreted the results based on Robert Merton's classic contributions to the sociology of science about the normative structure that regulates scientific activity. Our findings suggest that demanding institutional environments lead to varied scientific practices for academic production. Most researchers perceive that both locally produced and foreign-produced knowledge is used in their disciplinary fields, but in all disciplines a part of the scientific community perceives practices that are in tension with Merton's scientific norms, such as being more interested in acquiring personal fame than expanding the knowledge base, committing plagiarism, intentionally fragmenting research in order to publish more papers, and playing the role of evaluator anchored in personal beliefs and opinions rather than universal values for the production of scientific knowledge. This has implications for the training of scientists at a graduate level who participate in specialized practices and are learning to write and publish in specialized textual genres such as research articles.

Key words: disciplinary communities - scientific literacy - scientific ethos - roles - scientific practices.

\section{Introducción}

La publicación de artículos científicos es una de las tareas principales de los investigadores y resulta una actividad sustantiva de la tarea educativa. Los estudiantes de posgrado, al formarse como investigadores en sus respectivas áreas de especialidad, reconocen las formas de concebir la comunicación de la ciencia presentes en sus comunidades disciplinares a través de las prácticas de los investigadores expertos que desempeñan distintos roles asociados a la producción científica.

Entre las actividades realizadas en el trabajo científico, los roles de revisor y editor se suman al rol de autor (Carrasco, Kent, Keranen, 2012). La publicación en el campo científico es una actividad decisiva para demostrar la inclusión del investigador a una comunidad conformada por revisores, editores de revistas, autores y lectores (Englander, López-Bonilla, 2011).

En el nivel de posgrado, los estudiantes se forman participando en las prácticas de los especialistas. De acuerdo con Montes Silva y López Bonilla (2017), la participación de los novatos en las prácticas de los especialistas se refiere a una perspectiva vinculada a la investigación y la 
enseñanza de la literacidad disciplinar, que pretende que los estudiantes sepan cómo se concibe el conocimiento, cómo se investiga y cómo se comunica en cada disciplina, a fin de que en las etapas iniciales de su formación les resulte posible navegar por las distintas disciplinas de su currículo. Estudiar las prácticas científicas asociadas a la publicación, percibidas por los investigadores como miembros de su comunidad disciplinar, puede aportar elementos para entender algunos retos de la formación de los novatos desde el posgrado.

Este trabajo adopta una concepción de la literacidad disciplinar entendida como práctica sociocultural. El concepto de literacidad representa aquellas prácticas culturales de lectura y escritura situadas social, histórica e institucionalmente y, por ende, la literacidad es comprendida como una práctica social y un recurso colectivo integrado a un contexto específico (Barton, Hamilton, 2004; Barton, Hamilton, Ivanic, 2000). La literacidad disciplinar se refiere a un tipo especializado de literacidad académica (Montes Silva, López Bonilla, 2017) y como afirma Zavala (2008), exige reconocer el propósito social específico que requiere emplear la lectura y la escritura de una manera particular, en especial, si concierne a contextos de formación disciplinar más concentrados en desarrollar de forma planeada la capacidad de los estudiantes para participar en prácticas específicas de la disciplina (Fang, Coatoam, 2013).

De la distinción entre literacidad académica, referida a las prácticas más generalizables, y literacidad disciplinar, como las prácticas especializadas que Montes Silva y López Bonilla (2007) proponen, interesa situar a la publicación como un trabajo que se rige por el marco normativo de cada comunidad disciplinar y es socialmente construido en prácticas situadas que también están ligadas, en un sentido más amplio, a un quehacer científico que se realiza en el marco de los sistemas institucionales.

Las prácticas de publicación de los investigadores, ya sea de modo intencional o incluso no planeado, inciden en las prácticas de literacidad de los estudiantes, específicamente cuando se les está preparando para escribir y publicar géneros textuales especializados como el artículo de investigación. Como aprendices de científicos, al participar gradualmente en procesos de investigación los estudiantes juegan roles de autor, lector, crítico, editor y experto local (Carrasco Altamirano, Méndez Ochaita, Brambila Limón, Encinas Prudencio, Sánchez Hernández, 2020) y llegan a publicar como autores principales los resultados de sus investigaciones de tesis, incluso los primeros hallazgos, y en otros casos, también pueden llegar a figurar como coautores en las publicaciones de sus tutores.

Este trabajo analiza las percepciones expresadas por investigadores de una universidad pública mexicana sobre la tarea de publicación en los campos disciplinares, las cuales son interpretadas con nociones fundamentales de la sociología clásica de la ciencia de Merton y con bases en la sociología institucional. Particularmente, se busca dar cuenta de las prácticas de publicación que los investigadores de una institución de educación superior perciben en sus comunidades disciplinares. Se pretende aportar algunas reflexiones para pensar la formación 
de aprendices de científico como resultado de actividades situadas que les ofrecen oportunidades para observar y ejercer roles de actuación diversos.

\section{Marco de referencia y objetivos de la investigación}

La investigación que dio origen a este trabajo está anclada en la sociología clásica de la ciencia. Aquí se busca articular este enfoque con los estudios de literacidad disciplinar desde el punto de vista de las prácticas sociales. Es decir, el análisis parte del contexto social e institucional donde se llevan a cabo las prácticas científicas relacionadas con la comunicación de resultados de investigación, para revisar cómo se están percibiendo estas prácticas asociadas a la publicación científica por los miembros de las comunidades disciplinares de una universidad pública, a fin de que estos hallazgos puedan contribuir a pensar algunos retos asociados a esta temática.

En la sociología de la ciencia se reconoce, siguiendo los planteamientos de R. Merton, la existencia de marcos normativos que regulan la actividad de la ciencia en contextos sociales e institucionales, específicamente, que regulan el quehacer de los científicos en las instituciones y en determinadas circunstancias operan como un respaldo para asegurar la autonomía del campo científico.

Si bien existen normas de participación reconocidas por la comunidad científica (Merton, 1970, 2002), en trabajos anteriores (Méndez Ochaita, Romero Muñoz, Carrasco Altamirano, Kent Serna, Brambila Limón, 2020) se ha mostrado la importancia de analizar tanto las normas como las contranomas de las expresiones contemporáneas de las prácticas de investigadores consolidados. Aquí se resalta la expresión de tres binomios de normas y contranormas asociados a las tareas de producción y comunicación del conocimiento científico: universalismo-particularismo, interés-desinterés y escepticismo organizado-dogmatismo organizado (Merton, 1970, 2002). A continuación, se presentan las definiciones teóricas que nos aporta esta perspectiva:

El universalismo encuentra su expresión inmediata en el canon de que los títulos a la verdad, cualquiera que sea su fuente, tienen que ser sometidos a criterios impersonales preestablecidos: consonantes con las observaciones y con los conocimientos previamente confirmados (Merton, 2002: 639).

Sobre el desinterés, Merton (2002: 644) explica que "la ciencia, como ocurre con las profesiones en general, incluye desinterés como elemento institucional básico. No debe considerarse el desinterés igual al altruismo ni la acción interesada igual al egoísmo." y aclara:

Es más bien un tipo distintivo de control de un amplio margen de móviles lo que caracteriza la conducta de los científicos [...] La traducción de la norma del desinterés en práctica está apoyada eficazmente por la rendición de cuentas definitiva de los científicos ante sus compañeros (Merton, 2002: 645). 
El escepticismo organizado se interrelaciona de diversas maneras con los otros elementos del ethos científico. Es un mandato a la vez metodológico e institucional. La suspensión de juicio hasta que "estén a mano los hechos" y el escrutinio imparcial de las creencias de acuerdo con criterios empíricos y lógicos (Merton, 2002: 646).

Para Merton (2002), el ethos de la ciencia moderna, aunque no sea codificado, puede ser inferido del consenso de los científicos expresado en las prácticas instituidas sobre todo por usos y costumbres, pero también por vocaciones científicas que demuestran su compromiso con el conocimiento y desaprueban contravenciones a este ethos.

De acuerdo con Weingart $(2001,2015)$, el ethos de la ciencia rara vez se hace explícito, pero reconoce que su formulación más sucinta e influyente fue la desarrollada por Merton. Turner (2007) argumenta que la propuesta de Merton precisamente es de carácter normativo, porque corresponde a un periodo de intensa actividad política de la ciencia y responde a dicho contexto.

De algún modo, la expresión de estas normas en la actuación de los investigadores interesa porque actualmente podría tener una incidencia significativa en la formación de los aprendices de científicos.

Si bien Merton formuló su teoría normativa de la ciencia hace más de siete décadas (Merton, 1970, 2002), existe literatura reciente que retoma sus planteamientos en los debates contemporáneos sobre la actividad científica y los cambios en las estructuras normativas de la ciencia. Se ubican los estudios empíricos de Bieliński y Tomczyńska (2019), Kim y Kim (2018) y Macfarlane y Cheng (2008), que revisan la vigencia de un conjunto de normas y valores a partir de Merton, en la estructura científica actual de Polonia, Corea y Reino Unido, respectivamente. Estos estudios concuerdan en que las presiones crecientes sobre la vida académica pueden tener un impacto en la configuración de las normas o la emergencia de otras. Kønig, Børsen y Emmeche (2017), a través de un análisis documental, estudian cómo las estructuras de normas y valores se presuponen en prescripciones, proscripciones y objetivos para las prácticas de asesoramiento científico, identificando una pluralidad de esquemas normativos, característicos de los contextos contemporáneos complejos donde se lleva a cabo la actividad científica. Lam (2010) también sugiere que los científicos académicos son agentes activos que están cada vez más interesados en responder a las demandas del mercado y muestran una gran diversidad en sus orientaciones laborales.

Desde la perspectiva de las ciencias sociales, el estudio de Bucchi (2015) revisa teóricamente las normas y los valores de la ciencia a la luz de los cambios que la organización científica ha tenido en las últimas décadas, y se concentra en revisar aspectos como la subsistencia del concepto de comunidad científica de frente a la alta competencia que caracteriza al campo científico, asociada al logro de reputación y visibilidad de los científicos. Por su parte, Huutoniemi (2015) aborda la evolución de la revisión por pares, analizando los diversos modos de tal prác- 
tica como ejemplos de la norma del escepticismo organizado, y sostiene que los cambios institucionales recientes en la producción y evaluación del conocimiento implican una ambigüedad creciente en los propósitos y beneficiarios de la revisión por pares.

Por otra parte, Kalleberg $(2007,2015)$ aborda el debate actual que se ha generado en torno al tema normativo de la ciencia, y apunta que el sistema tradicional de autoregulación que se sigue en la investigación, relacionado con el compromiso de verdad de la ciencia, actualmente se cultiva y supervisa de manera más explícita, complementado con procedimientos y comités en niveles superiores como a nivel de organizaciones. También se refiere al fraude en la investigación de tres modos: la fabricación de datos ficticios, la falsificación de datos y métodos, y el fenómeno del plagio, para centrarse en el surgimiento histórico de la ética de la investigación moderna, revisando para ello los planteamientos de Merton.

La relevancia de este artículo es que, en el marco de estos estudios referidos, aporta elementos para revisar algunas de las prácticas de publicación de científicos de distintas disciplinas, a través de la percepción de los miembros de las comunidades disciplinares en una universidad estatal mexicana. De esta aproximación habrá que profundizar sobre lo que se devela en este trabajo sobre la diversidad de prácticas de publicación en las disciplinas, donde también participan estudiantes que son aprendices de científicos desde el posgrado.

\section{Perspectivas teóricas}

En general, en los marcos institucionales de las universidades, la actividad científica se organiza por campos disciplinares y está relacionada con las tareas de docencia, investigación, innovación y/o desarrollo tecnológico. Si bien en esta forma de organización departamental o por áreas se representan, cultivan y desarrollan las disciplinas, y las tareas interrelacionadas evidentemente complejizan el estudio de las prácticas en las disciplinas, sucede lo mismo con las prácticas de publicación.

Las estructuras organizacionales delimitan de cierto modo las áreas disciplinares en las instituciones de educación superior, por ser espacios institucionales bastante definidos van configurando ámbitos de participación cotidiana de científicos y de estudiantes en formación. De acuerdo con Becher y Trowler (2001) y con Merton (2002), los investigadores comparten ciertas normas que los motivan y les regulan como actores en sus ámbitos disciplinares e institucionales (Méndez Ochaita, Romero Muñoz, Carrasco Altamirano, Kent Serna, Brambila Limón, 2020). Estas normas y valores que son compartidos en las comunidades disciplinares, sin duda influyen en la formación profesional de los estudiantes, sobre todo, de los aprendices de científicos que se están formando como investigadores.

Desde este punto de vista y siguiendo las bases generales que ofrece la sociología de la ciencia y la sociología institucional (Fernández Esquinas, Torres Albero, 2009; Merton, 1970, 2002; Merton, Storer, 1979), cabe subrayar que, si bien la ciencia se concibe como una institu- 
ción social, puede reconocerse que en el marco del sistema nacional de ciencia y tecnología también se establece como un espacio de luchas para obtener recursos y posicionamientos atendiendo a jerarquías, prestigios, intereses y capitales (Bourdieu, 1976).

Las diversas actividades de los investigadores responden a las estructuras normativas de la ciencia y funcionan también como guías en la construcción de identidad en su actuación como científicos, lo mismo ocurre para estudiantes que se forman como parte de una comunidad disciplinar. Es decir, las normas y los valores que comparten los científicos en sus prácticas cotidianas, al producir conocimiento científico y al emplear determinados métodos, también constituyen una normativa que orienta las prácticas disciplinares de los aprendices, incluidas las que están relacionadas con la escritura y la publicación de géneros especializados como el artículo de investigación.

También es importante resaltar una dimensión ideológica de la escritura disciplinar como práctica social, puesto que implica relaciones de poder que están fuertemente institucionalizadas y se manifiestan de diversas formas, tanto en las actividades como en el discurso escrito en cada disciplina (Montes Silva, López Bonilla, 2017). Cada grupo disciplinar tiene participación en las prácticas científicas que se rigen por reglas y convenciones creadas y alimentadas por los actores disciplinares como miembros acreditados de grupos del sistema de ciencia y tecnología que certifica posiciones, jerarquías y prestigios.

En un trabajo previo (Carrasco Altamirano, Méndez Ochaita, Brambila Limón, Encinas Prudencio, Sánchez Hernández, 2020) se presentó una caracterización de los distintos roles que los aprendices de científicos deben realizar, y para esta revisión se retoman dos de ellos, la autoría y la dictaminación de trabajos de otros, por estar relacionados con las prácticas de literacidad. En dicho trabajo se señaló que los científicos en formación experimentan la ciencia como una profesión y abrazan los valores de esta actividad. Aquí se reitera que la comunidad disciplinar valida la participación y el reconocimiento de cada uno de sus miembros, novatos y expertos. Y siguiendo a Delamont y Atkinson (2001), se apunta que los novatos aprenden tácitamente, es decir, que las actividades de enseñanza no siempre están formalmente codificadas. En otras palabras, los aprendizajes logrados por ellos en parte "son típicamente habilidades y formas de conocimiento tácitos que de forma progresiva se incorporan como habitus" (Carrasco Altamirano, Méndez Ochaita, Brambila Limón, Encinas Prudencio, Sánchez Hernández, 2020: 35).

\section{Metodología}

El enfoque metodológico de este estudio es cualitativo. Como se ha mencionado anteriormente, este trabajo se deriva de una investigación iniciada en 2018 sobre la vigencia de las normas científicas mertonianas en la universidad contemporánea y sus expresiones en las prácticas científicas. Para dicha investigación, se aplicó una encuesta en línea a los investigadores de distintos campos disciplinares de una universidad pública mexicana. 
El instrumento, aplicado en línea, consta de 41 ítems. A través de este se recolectaron las percepciones de los encuestados acerca de las normas y las prácticas típicas de la actividad científica en sus campos disciplinares (28 ítems), los datos generales de los participantes (13 ítems), tales como su edad y género, información general sobre su formación disciplinar, su experiencia en investigación y la unidad de adscripción. El presente trabajo reporta el material empírico de seis ítems que se refieren a las prácticas de publicación percibidos por los investigadores en sus campos disciplinares (véanse las tablas 1 y 2 ).

Los encuestados registraron sus percepciones respondiendo a una escala Likert de cinco opciones que indican el grado de acuerdo o desacuerdo con lo expresado en cada ítem, permitiendo también el registro de una postura neutral sobre el contenido de lo enunciado.

La fiabilidad interna de la escala de medida del instrumento se calculó con el índice de alfa de Cronbach en 0.602 para los 28 ítems que miden las percepciones de los investigadores (valor aceptable para un estudio exploratorio). Este índice expresa el grado de homogeneidad que tiene la escala para medir el constructo entre 0 y 1.

También se calcularon las frecuencias y los porcentajes de respuesta por cada ítem de percepción con la herramienta de análisis Statical Package for the Social Science (SPSS) (Paquete Estadístico para las Ciencias Sociales). Los 28 ítems de percepciones se agruparon en constructos referidos a las normas, contranormas, prácticas y valores científicos, y se ordenaron en tablas cruzadas por: división de estudios superiores (DES), rango de edad y género. Cabe aclarar que las DES se refieren a la organización en la universidad por área de conocimiento de sus unidades: escuelas, facultades e institutos.

\section{Características de la muestra}

La muestra está conformada por 352 investigadores que respondieron a la encuesta, que corresponde a $40 \%$ de la población considerada para el estudio $(\mathrm{N}=873)$ : los integrantes del padrón institucional de investigadores en 2018. Esta muestra es representativa con un nivel de confianza estadística de $95 \%$, con un intervalo de confianza de 4, estimado para una variabilidad máxima de $50 \%$ por tratarse de una población heterogénea.

A continuación se presentan dos gráficas para caracterizar la muestra. La gráfica 1 presenta la distribución de los encuestados por género y lugar de adscripción en la universidad, y la gráfica 2 presenta la misma distribución por grupos de edad. En ambos casos se señala que hay una distribución variada de los participantes de la encuesta por campos disciplinares, género y rangos de edad. 
Gráfica 1. Adscripción de los investigadores por División de Estudios Superiores

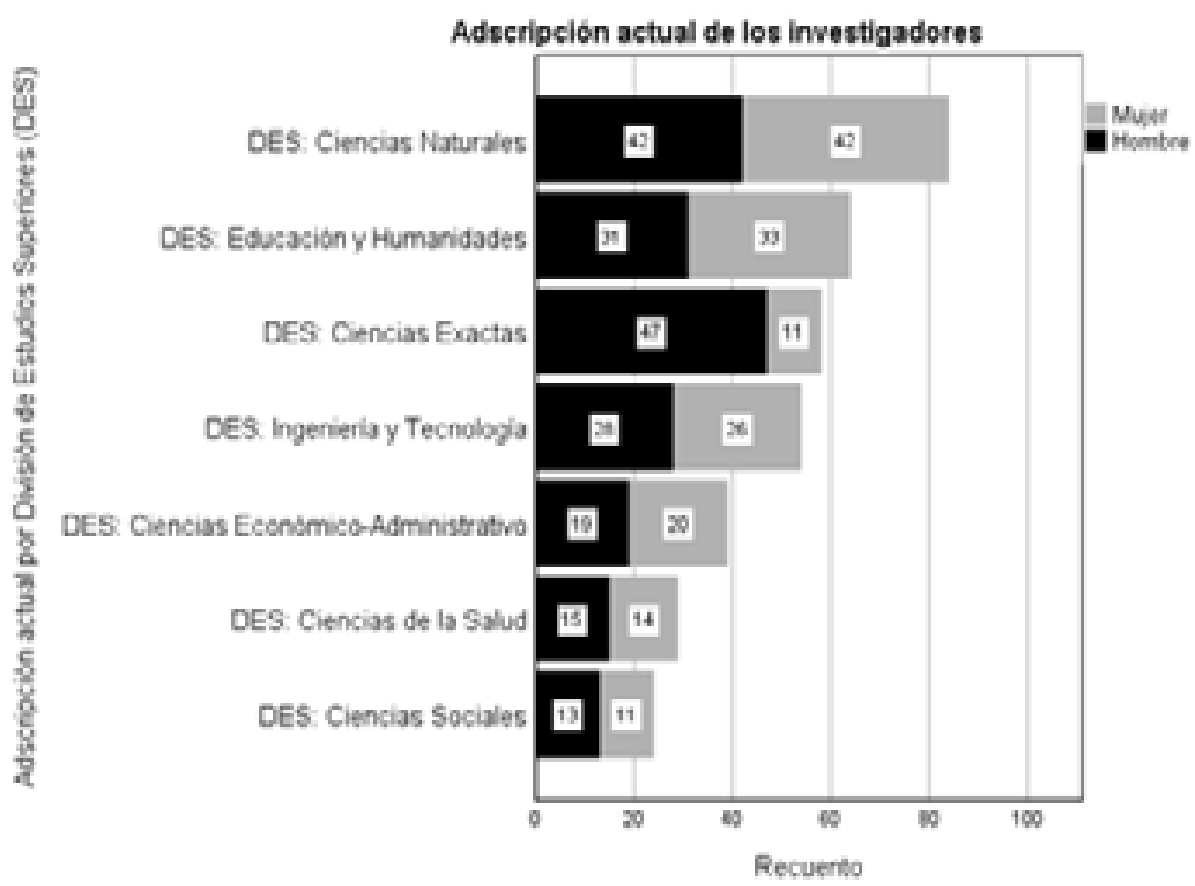

Gráfica 2. Adscripción actual de los investigadores por grupos de edad

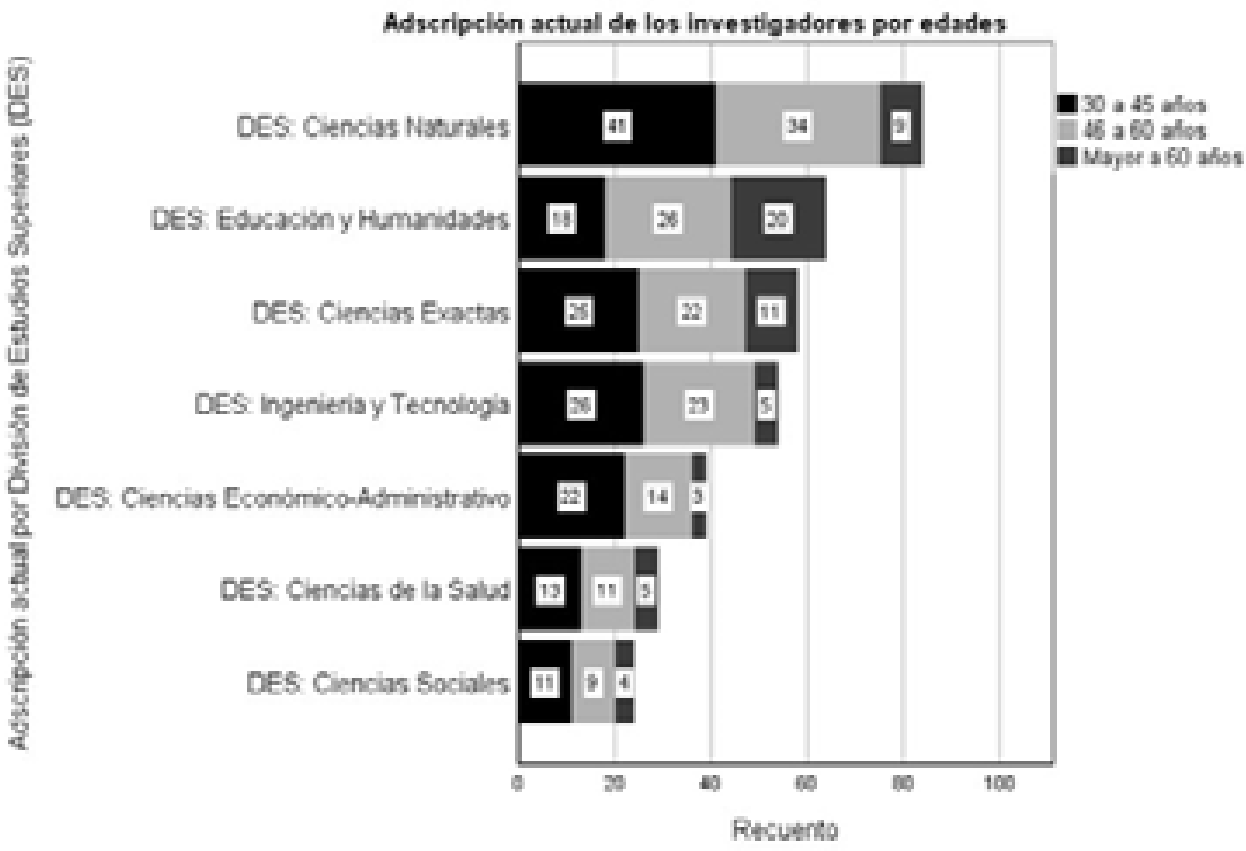


Como puede estimarse con los datos de la gráfica 1, la muestra se compone por 157 mujeres que representan $45 \%$ de los encuestados y por 195 hombres que representan 55\%. Y de acuerdo con los datos mostrados en la gráfica 2, a la encuesta respondieron 156 investigadores jóvenes, con edades entre 30 y 45 años, 139 con edades intermedias entre 46 a 60 años y 57 con edades mayores a 60 años. De los encuestados, $74 \%$ pertenece a los campos de las ciencias naturales y exactas, educación y humanidades e ingeniería y tecnología.

Si bien las tres variables: DES, género y rango de edad, representan grupos diferentes, a través de la prueba de igualdad de medias de grupos de Lambda de Wilks se determinó que no hay diferencias entre las varianzas de los grupos a nivel estadístico. Los coeficientes obtenidos son 0.949 para las DES y valores mayores a 0.99 para las demás variables con valores de significancia mayores a $5 \%(p>0.05)$.

Debido al enfoque cualitativo del presente trabajo, el análisis destaca solo las diferencias disciplinares, aunque no hay diferencias significativas a nivel estadístico.

\section{Análisis de resultados}

Se presentan las percepciones sobre las prácticas de publicación de quienes, entre sus distintos roles en una institución de educación pública mexicana, no solo producen conocimiento al publicar y certifican el valor del trabajo de otros autores, sino también forman a los nuevos cuadros en sus campos disciplinares (véanse tablas 1 y 2 ). 
Tabla 1. Percepciones sobre prácticas de publicación relacionadas con normas mertonianas

\begin{tabular}{|c|c|c|c|c|c|c|c|c|c|}
\hline \multirow[b]{2}{*}{ Ítem y norma relacionada } & & \multicolumn{7}{|c|}{ División de Estudios Superiores (DES) } & \multirow[b]{2}{*}{ Total } \\
\hline & & $\begin{array}{c}\text { Ciencias de } \\
\text { la Salud }\end{array}$ & $\begin{array}{c}\text { Ciencias } \\
\text { Económico- } \\
\text { Administrativo }\end{array}$ & $\begin{array}{c}\text { Ciencias } \\
\text { Exactas }\end{array}$ & $\begin{array}{c}\text { Ciencias } \\
\text { Naturales }\end{array}$ & $\begin{array}{l}\text { Ciencias } \\
\text { Sociales }\end{array}$ & $\begin{array}{c}\text { Educación y } \\
\text { Humanidades }\end{array}$ & $\begin{array}{c}\text { Ingeniería y } \\
\text { Tecnología }\end{array}$ & \\
\hline \multirow{6}{*}{$\begin{array}{l}\text { Conoce investigadores que } \\
\text { aprovechan y citan la } \\
\text { producción local y nacional } \\
\text { de la misma manera que el } \\
\text { conocimiento producido en } \\
\text { otros países. }\end{array}$} & Acuerdo & 16 & 28 & 35 & 57 & 13 & 40 & 31 & 220 \\
\hline & & $55.2 \%$ & $71.8 \%$ & $60.3 \%$ & $67.9 \%$ & $54.2 \%$ & $62.5 \%$ & $57.4 \%$ & $62.5 \%$ \\
\hline & Neutro & 4 & 8 & 11 & 14 & 4 & 10 & 14 & 65 \\
\hline & & $13.8 \%$ & $20.5 \%$ & $19.0 \%$ & $16.7 \%$ & $16.7 \%$ & $15.6 \%$ & $25.9 \%$ & $18.5 \%$ \\
\hline & Desacuerdo & 9 & 3 & 12 & 13 & 7 & 14 & 9 & 67 \\
\hline & & $31.0 \%$ & $7.7 \%$ & $20.7 \%$ & $15.5 \%$ & $29.2 \%$ & $21.9 \%$ & $16.7 \%$ & $19.0 \%$ \\
\hline \multirow[t]{2}{*}{ Universalismo } & Total & 29 & 39 & 58 & 84 & 24 & 64 & 54 & 352 \\
\hline & & $100.0 \%$ & $100.0 \%$ & $100.0 \%$ & $100.0 \%$ & $100.0 \%$ & $100.0 \%$ & $100.0 \%$ & $100.0 \%$ \\
\hline \multirow{6}{*}{$\begin{array}{l}\text { En las revistas científicas } \\
\text { de su campo los } \\
\text { dictaminadores juzgan los } \\
\text { conocimientos con } \\
\text { independencia de sus } \\
\text { creencias u opiniones }\end{array}$} & Acuerdo & 16 & 22 & 40 & 58 & 9 & 32 & 36 & 213 \\
\hline & & $55.2 \%$ & $56.4 \%$ & $69.0 \%$ & $69.0 \%$ & $37.5 \%$ & $50.0 \%$ & $66.7 \%$ & $60.5 \%$ \\
\hline & Neutro & 6 & 4 & 9 & 14 & 7 & 11 & 8 & 59 \\
\hline & & $20.7 \%$ & $10.3 \%$ & $15.5 \%$ & $16.7 \%$ & $29.2 \%$ & $17.2 \%$ & $14.8 \%$ & $16.8 \%$ \\
\hline & Desacuerdo & 7 & 13 & 9 & 12 & 8 & 21 & 10 & 80 \\
\hline & & $24.1 \%$ & $33.3 \%$ & $15.5 \%$ & $14.3 \%$ & $33.3 \%$ & $32.8 \%$ & $18.5 \%$ & $22.7 \%$ \\
\hline \multirow[t]{2}{*}{ Escepticismo organizado } & Total & 29 & 39 & 58 & 84 & 24 & 64 & 54 & 352 \\
\hline & & $100.0 \%$ & $100.0 \%$ & $100.0 \%$ & $100.0 \%$ & $100.0 \%$ & $100.0 \%$ & $100.0 \%$ & $100.0 \%$ \\
\hline \multirow{6}{*}{$\begin{array}{l}\text { Los investigadores } \\
\text { fragmentan la publicación } \\
\text { de un hallazgo con la sola } \\
\text { intención de tener varias } \\
\text { publicaciones. }\end{array}$} & Acuerdo & 19 & 24 & 26 & 46 & 17 & 39 & 27 & 198 \\
\hline & & $65.5 \%$ & $61.5 \%$ & $44.8 \%$ & $54.8 \%$ & $70.8 \%$ & $60.9 \%$ & $50.0 \%$ & $56.3 \%$ \\
\hline & Neutro & 6 & 10 & 16 & 15 & 3 & 19 & 18 & 87 \\
\hline & & $20.7 \%$ & $25.6 \%$ & $27.6 \%$ & $17.9 \%$ & $12.5 \%$ & $29.7 \%$ & $33.3 \%$ & $24.7 \%$ \\
\hline & Desacuerdo & 4 & 5 & 16 & 23 & 4 & 6 & 9 & 67 \\
\hline & & $13.8 \%$ & $12.8 \%$ & $27.6 \%$ & $27.4 \%$ & $16.7 \%$ & $9.4 \%$ & $16.7 \%$ & $19.0 \%$ \\
\hline \multirow[t]{2}{*}{ Desinterés } & Total & 29 & 39 & 58 & 84 & 24 & 64 & 54 & 352 \\
\hline & & $100.0 \%$ & $100.0 \%$ & $100.0 \%$ & $100.0 \%$ & $100.0 \%$ & $100.0 \%$ & $100.0 \%$ & $100.0 \%$ \\
\hline \multirow{6}{*}{$\begin{array}{l}\text { En su campo, cada vez hay } \\
\text { más científicos interesados } \\
\text { en adquirir fama personal } \\
\text { que en ampliar la base del } \\
\text { conocimiento científico. }\end{array}$} & Acuerdo & 17 & 17 & 24 & 36 & 12 & 39 & 23 & 168 \\
\hline & & $58.6 \%$ & $43.6 \%$ & $41.4 \%$ & $42.9 \%$ & $50.0 \%$ & $60.9 \%$ & $42.6 \%$ & $47.7 \%$ \\
\hline & Neutro & 6 & 10 & 14 & 25 & 4 & 14 & 16 & 89 \\
\hline & & $20.7 \%$ & $25.6 \%$ & $24.1 \%$ & $29.8 \%$ & $16.7 \%$ & $21.9 \%$ & $29.6 \%$ & $25.3 \%$ \\
\hline & Desacuerdo & 6 & 12 & 20 & 23 & 8 & 11 & 15 & 95 \\
\hline & & $20.7 \%$ & $30.8 \%$ & $34.5 \%$ & $27.4 \%$ & $33.3 \%$ & $17.2 \%$ & $27.8 \%$ & $27.0 \%$ \\
\hline \multirow[t]{2}{*}{ Desinterés } & Total & 29 & 39 & 58 & 84 & 24 & 64 & 54 & 352 \\
\hline & & $100.0 \%$ & $100.0 \%$ & $100.0 \%$ & $100.0 \%$ & $100.0 \%$ & $100.0 \%$ & $100.0 \%$ & $100.0 \%$ \\
\hline
\end{tabular}

Fuente: elaboración propia.

Formarse como investigadores exige aprender a participar como miembros de comunidades científicas, y ello plantea el reto de escribir artículos que den cuenta de resultados de investigación y publicarlos. La producción local de conocimiento científico, así como la producción extranjera, son igualmente valoradas. Más de la mitad de los investigadores encuestados percibe que en sus disciplinas se aprovecha tanto el conocimiento producido localmente como el de otras latitudes (62.5\%), pero el porcentaje disminuye en algunas áreas como ciencias sociales y de la salud, y se eleva hasta $71.8 \%$ en el área económico-administrativa. Este ítem está relacionado con la norma mertoniana del universalismo.

Una tendencia similar (60.5\% de acuerdo) se observa en las percepciones sobre la imparcialidad que se espera en la dictaminación del conocimiento científico, con algunas variantes disciplinares. 
Ser autor es un rol de la membresía científica, que también se expresa en la actuación en otros roles como el de revisor o cuidador de frontera (Carrasco Altamirano, Méndez Ochaita, Brambila Limón, Encinas Prudencio, Sánchez Hernández, 2020) y contribuye a la construcción disciplinar del conocimiento científico. Una expresión de escepticismo organizado a favor de la circulación del conocimiento científico sería la realización profesional de dictámenes de los trabajos de otros investigadores sin que en estos juicios interfieran las creencias y opiniones personales de los revisores, es decir, que la valoración del conocimiento científico se realice con criterios impersonales, verificables a través de la lógica y el sustento empírico según el método que siga la investigación.

La independencia de creencias y opiniones personales al evaluar los trabajos de los colegas, aunque es un valor reconocido por actores de diversas áreas disciplinares, resulta menos perceptible para los científicos sociales pues ellos reconocen que los dictaminadores de su disciplina actúan con menos independencia, solo 37.5\% de ellos admite la aplicación de criterios impersonales en la dictaminación de contribuciones para publicar. Por otra parte, 69\% de los investigadores de las ciencias naturales y de las exactas perciben que los dictaminadores de sus respectivas áreas disciplinares actúan con mayor independencia, siendo estas disciplinas las que más se aproximan a esta expresión normativa de escepticismo organizado.

El desinterés es la norma que valora la motivación por ampliar el conocimiento científico y la comunicación completa de una investigación sobre el deseo de adquirir fama personal. En la investigación, fragmentar el saber para cumplir con exigencias sectoriales de contar con un número determinado de publicaciones es un comportamiento menos percibido en las ciencias exactas (44.8\%) y más percibido en las ciencias sociales (70.8\%). Las diferencias disciplinares son evidentes, pero hacen falta más estudios para explicar este fenómeno. Es posible pensar que la partición de una investigación en ciencias exactas es menos factible que en el caso de los estudios sociales, dada la complejidad de las relaciones sociales y la amplitud de perspectivas e interpretaciones, podría publicarse más de un artículo derivado de una sola investigación.

Adquirir fama personal como principal motivación, antes que ampliar la base del conocimiento científico, también es una intención que se opone al desinterés del científico. En las ciencias exactas hay menos interés en adquirir fama personal (41.4\%), mientras que en ciencias de la salud, educación y humanidades la motivación de adquirir reputación personal es mayor (58.6 y 60.9\% respectivamente).

En la tabla 2 se presentan dos ítems que, sin estar asociados explícitamente a las normas de Merton, matizan su expresión porque muestran que $39.5 \%$ de los encuestados en todas las disciplinas reconocen el fenómeno del plagio. Los investigadores de ciencias sociales perciben más este fenómeno y los de ciencias exactas y naturales menos, lo que pone de manifiesto, nuevamente, algunas variaciones disciplinares. 
Por otra parte, $62.5 \%$ de los encuestados considera que las condiciones institucionales que organizan el desarrollo de la ciencia ejercen presiones por competir que pueden inducir comportamientos pocos éticos.

Tabla 2. Percepción sobre prácticas de publicación en los campos disciplinares

\begin{tabular}{|c|c|c|c|c|c|c|c|c|c|}
\hline \multirow[b]{3}{*}{ Ítem } & & \multicolumn{7}{|c|}{ División de Estudios Superiores (DES) } & \multirow[b]{3}{*}{ Total } \\
\hline & & \multicolumn{3}{|c|}{ Ciencias } & \multirow[b]{2}{*}{$\begin{array}{c}\text { Ciencias } \\
\text { Naturales }\end{array}$} & \multirow[b]{2}{*}{$\begin{array}{l}\text { Ciencias } \\
\text { Sociales }\end{array}$} & \multirow[b]{2}{*}{$\begin{array}{c}\text { Educación y } \\
\text { Humanidades }\end{array}$} & \multirow[b]{2}{*}{$\begin{array}{c}\text { Ingeniería y } \\
\text { Tecnología }\end{array}$} & \\
\hline & & $\begin{array}{c}\text { Ciencias de la } \\
\text { Salud }\end{array}$ & $\begin{array}{c}\text { Económico- } \\
\text { Administrativo }\end{array}$ & $\begin{array}{l}\text { Ciencias } \\
\text { Exactas }\end{array}$ & & & & & \\
\hline \multirow{8}{*}{$\begin{array}{l}\text { Sabe de investigadores } \\
\text { que utilizan aportes de } \\
\text { otros científicos sin } \\
\text { citarlos apropiadamente }\end{array}$} & Acuerdo & 15 & 19 & 18 & 26 & 13 & 30 & 18 & 139 \\
\hline & & $51.7 \%$ & $48.7 \%$ & $31.0 \%$ & $31.0 \%$ & $54.2 \%$ & $46.9 \%$ & $33.3 \%$ & $39.5 \%$ \\
\hline & Neutro & 7 & 9 & 13 & 32 & 5 & 22 & 17 & 105 \\
\hline & & $24.1 \%$ & $23.1 \%$ & $22.4 \%$ & $38.1 \%$ & $20.8 \%$ & $34.4 \%$ & $31.5 \%$ & $29.8 \%$ \\
\hline & Desacuerdo & 7 & 11 & 27 & 26 & 6 & 12 & 19 & 108 \\
\hline & & $24.1 \%$ & $28.2 \%$ & $46.6 \%$ & $31.0 \%$ & $25.0 \%$ & $18.8 \%$ & $35.2 \%$ & $30.7 \%$ \\
\hline & Total & 29 & 39 & 58 & 84 & 24 & 64 & 54 & 352 \\
\hline & & $100.0 \%$ & $100.0 \%$ & $100.0 \%$ & $100.0 \%$ & $100.0 \%$ & $100.0 \%$ & $100.0 \%$ & $100.0 \%$ \\
\hline \multirow{8}{*}{$\begin{array}{l}\text { Las presiones por } \\
\text { competir inducen } \\
\text { comportamientos poco } \\
\text { éticos entre los } \\
\text { investigadores de su } \\
\text { campo }\end{array}$} & Acuerdo & 18 & 25 & 35 & 45 & 19 & 49 & 29 & 220 \\
\hline & & $62.1 \%$ & $64.1 \%$ & $60.3 \%$ & $53.6 \%$ & $79.2 \%$ & $76.6 \%$ & $53.7 \%$ & $62.5 \%$ \\
\hline & Neutro & 9 & 7 & 17 & 18 & 4 & 7 & 14 & 76 \\
\hline & & $31.0 \%$ & $17.9 \%$ & $29.3 \%$ & $21.4 \%$ & $16.7 \%$ & $10.9 \%$ & $25.9 \%$ & $21.6 \%$ \\
\hline & Desacuerdo & 2 & 7 & 6 & 21 & 1 & 8 & 11 & 56 \\
\hline & & $6.9 \%$ & $17.9 \%$ & $10.3 \%$ & $25.0 \%$ & $4.2 \%$ & $12.5 \%$ & $20.4 \%$ & $15.9 \%$ \\
\hline & Total & 29 & 39 & 58 & 84 & 24 & 64 & 54 & 352 \\
\hline & & $100.0 \%$ & $100.0 \%$ & $100.0 \%$ & $100.0 \%$ & $100.0 \%$ & $100.0 \%$ & $100.0 \%$ & $100.0 \%$ \\
\hline
\end{tabular}

Fuente: elaboración propia.

\section{Conclusiones}

Si bien este trabajo no es representativo de las percepciones de los científicos de un país, sí ejemplifica en el contexto contemporáneo de México algunas de las percepciones de los académicos que realizan trabajo de investigación en una universidad pública estatal que cuenta con un alto porcentaje de investigadores reconocidos por el Sistema Nacional de Investigadores (SNI) (69\% de la muestra conformada pertenece al SNI).

Dado que la investigación científica y el desarrollo tecnológico son actividades sustantivas de la labor de una universidad, indagar sobre las percepciones de la publicación y la escritura disciplinares es relevante debido a que es una práctica fundamental de los expertos $y$, al mismo tiempo, una tarea formativa de la educación superior a nivel de posgrado.

Los investigadores de las distintas comunidades disciplinares perciben de modo parecido las prácticas de publicación. Las diferencias disciplinares son pocas. En general, reconocen que la alta competencia en entornos de exigencia institucional de algún modo afecta el comportamiento de algunos miembros, expresándose, por ejemplo, en fenómenos como el plagio, problemática que los académicos perciben en la actuación de una parte de la comunidad. 
Los entornos institucionales de la ciencia y la tecnología en México son altamente competitivos y las exigencias de publicación cada vez son mayores. Como resultado de este estudio, a este escenario se suma que la participación de los investigadores de estas prácticas es diversa, algunos perciben que en sus comunidades disciplinares hay investigadores más interesados en la obtención de reconocimiento que en ampliar la base del conocimiento y que hay quienes dictaminan el trabajo científico tomando criterios personales, y no tanto por la evidencia lógica y empírica de las contribuciones.

En definitiva, estas condiciones disciplinares, interpretadas a través de las percepciones de los académicos, inciden en las prácticas de literacidad de las que participan los estudiantes y en las que derivan aprendizajes tácitos como investigadores que eventualmente llegarán a realizar sus propias prácticas de publicación cuando tengan que producir y revisar artículos de investigación. Si la fragmentación de resultados de investigación y las percepciones sobre plagio son reconocidas por investigadores de todas las disciplinas, sería conveniente en el futuro desarrollar estudios que den cuenta de casos concretos que repercuten en el desarrollo mismo de la investigación científica y en la formación de científicos.

Las percepciones de los académicos reflejan comportamientos que pudieran estar presentes en los equipos de investigación y, en este sentido, podrían constituirse en oportunidades de conocimientos tácitos para científicos novatos a quienes se forma, precisamente, participando de las actividades y encuentros de cada equipo. En estos eventos, los científicos en formación aprenden sin que las enseñanzas sean formal ni curricularmente organizadas. No obstante, si en estos eventos se manifestaran las tendencias presentadas en este trabajo a partir de las percepciones de los académicos, quizá se podría documentar una dosis de indiferencia en la actividad científica, además fincada en la contrariedad, pues tales actividades estarían asociadas a la construcción de las carreras académicas de los científicos expertos.

Los aprendizajes tácitos quizá sean los que contribuyan en el futuro a redefinir las percepciones encontradas en este trabajo, porque definitivamente los valores científicos tienen expresiones particulares en las prácticas de publicación como en la exigencia de membresía. Finalmente, también habría que considerar las posibilidades que ofrece la instrucción de la literacidad disciplinar sobre las prácticas de publicación. Quizás es momento de considerar la alfabetización disciplinar de modo explícito en el diseño curricular de los doctorados científicos en México.

\section{Referencias}

Barton, D.; M. Hamilton; R. Ivanic (2000). Situated Literacies: Reading and Writing in Context: Routledge.

Barton, D.; M. Hamilton (2004). La literacidad entendida como práctica social. Escritura y sociedad. Nuevas perspectivas teóricas y etnográficas, 109-139. 
Becher, T.; P. Trowler (2001). Tribus y territorios académicos. La indagación intelectual y las culturas de las disciplinas. Barcelona: Gedisa.

Bieliński, J.; A. Tomczyńska (2019). The Ethos of Science in Contemporary Poland. Minerva, 57(2), 151-173. https://doi.org/10.1007/s11024-018-9365-1

Bourdieu, P. (1976). El campo científico. Redes, Dossier, 131-160.

Bucchi, M. (2015). Norms, Competition and Visibility in Contemporary Science: The Legacy of Robert K. Merton. Journal of Classical Sociology, 15(3), 233-252.

Carrasco Altamirano, A.; M. F. Méndez Ochaita; R. Brambila Limón; F. Encinas Prudencio; V. Sánchez Hernández (2020). Leer y escribir como interpretación de roles, aprender de experiencias de estudiantes de doctorado. Didac, (75), 32-39.

Carrasco, A.; R. Kent; N. Keranen (2012). Learning Careers and Enculturation: Production of Scientific Papers by Phd Students in a Mexican Fhysiology Laboratory: An Exploratory Case Study. En C. Bazerman; C. Dean; J. Early; K. Lunsford; S. Null; P. Rogers; A. Stansell (Eds.). International Advances in Writing Research: Cultures, Places, Measures (pp. 335-352). Colorado, CO/Anderson, SC: The WAC Clearinghouse/Parlor Press.

Delamont, S.; P. Atkinson (2001). Doctoring Uncertainty. Social Studies of Science, 31(1), 87-107.

Englander, K.; G. López-Bonilla (2011). Acknowledging or Denying Membership: Reviewer Responses to Non-anglophone Scientists' Manuscripts. Discourse Studies, 134(4), 395416.

Fang, Z.; S. Coatoam (2013). Disciplinary Literacy: What You Want to Know About It. Journal of Adolescent \& Adult Literacy, 56(8), 627-632.

Fernández Esquinas, M.; C. Torres Albero (2009). La ciencia como institución social: clásicos y modernos institucionalismos en la sociología de la ciencia. Arbor: Ciencia, Pensamiento, Cultura, 185(738).

Huutoniemi, K. (2015). Peer Review: Organized Skepticism. En Wright, J. D. (ed.). International Encyclopedia of the Social \& Behavioral Sciences (Second Edition) (pp. 685-689). Oxford: Elsevier.

Kalleberg, R. (2007). A Reconstruction of the Ethos of Science. Journal of Classical Sociology, 7(2), 137-160.

Kalleberg, R. (2015). Scientific Misconduct, Plagiarism, and Institutional Control of Misconduct. En Wright, J. D. (Ed.). International Encyclopedia of the Social \& Behavioral Sciences (Second Edition). Oxford: Elsevier, 313-317.

Kim, S. Y.; Y. Kim (2018). The Ethos of Science and its Correlates: An Empirical Analysis of Scientists' Endorsement of Mertonian Norms. Science, Technology and Society, 23(1), 1-24.

Kønig, N.; T. Børsen; C. Emmeche (2017). The Ethos of Post-normal Science. Futures, (91), 12-24. Lam, A. (2010). From 'Ivory Tower Traditionalists' to 'Entrepreneurial Scientists'?: Academic Scientists in Fuzzy University -Industry Boundaries. Social Studies of Science, 40(2), 307-340. 
Macfarlane, B.; M. Cheng (2008). Communism, Universalism and Disinterestedness: Re-examining Contemporary Support among Academics for Merton's Scientific Norms. Journal of Academic Ethics, 6(1), 67-78.

Méndez Ochaita, M. F.; J. Romero Muñoz; A. Carrasco Altamirano; R. Kent Serna; R. Brambila Limón (2020). Normas y valores científicos: la ambivalencia percibida en las prácticas de investigación en una universidad pública mexicana. Sociológica, 98(34), 43-90.

Merton, R. K. (1970). The Normative Structure of Science. En Merton, R. K. (ed.). The Sociology of Science: Theoretical and Empirical Investigations. Chicago: University of Chicago Press.

Merton, R. K. (2002). Teoría y estructura sociales. México: Fondo de Cultura Económica.

Merton, R. K.; N. W. Storer (1979). The Sociology of Science: Theoretical and Empirical Investigations. Chicago: University of Chicago Press.

Montes Silva, M. E.; G. López Bonilla (2017). Literacidad y alfabetización disciplinar: enfoques teóricos y propuestas pedagógicas. Perfiles Educativos, 39(155), 162-178.

Turner, S. (2007). Merton's 'Norms' in Political and Intellectual Context. Journal of Classical Sociology, 7(2), 161-178.

Weingart, P. (2001). Norms in Science. En Smelser, N. J.; P. B. Baltes (eds.). International Encyclopedia of the Social \& Behavioral Sciences. Oxford: Pergamon, 10720-10723.

Weingart, P. (2015). Norms in Science. In J. D. Wright (Ed.). International Encyclopedia of the Social \& Behavioral Sciences (Second Edition). Oxford: Elsevier, 11-14.

Zavala, V. (2008). La literacidad, o lo que la gente "hace" con la lectura y la escritura. Textos de Didáctica de la Lengua y la Literatura, (47), 71-79. 